\title{
Deformation Mechanism of Polyethylene Spherulites as Observed by Wide-Angle Light Scattering
}

\author{
Akira Todo, ${ }^{*}$ Takeji Hashmoto, and Hiromichi KAwAI \\ Department of Polymer Chemistry, Faculty of Engineering, \\ Kyoto University, Kyoto 606, Japan.
}

(Received March 14, 1978)

\begin{abstract}
The deformation mechanism of polyethylene spherulites was studied by analyzing the change in wide angle "ring" scattering arising from the helicoidal twisting of lamellae with applied uniaxial stretching. It was verified by numerical calculations of the ring scattering that the scattering angles $\theta_{\max , \mu}$ giving rise to maximum ring intensity at azimuthal angle $\mu$ are independent of the polarization conditions $\left(V_{\mathrm{V}}, H_{\mathrm{V}}, H_{\mathrm{H}}\right.$, and etc.) and orientation of the optical axes of the scattering elements with respect to spherulite radii (i.e., independent of the internal reorientation processes of the optical axes within the deformed spherulites). These angles are dependent entirely on the ring spacing in a particular angular region of the spherulite in accordance with the Bragg's equation. Thus the studies of the ring spacing as a function of the azimuthal angles between the stretching direction and the scanning plane give information on angular dependence of the spherulite deformation. These results indicated, for a special polyethylene studied, that the spherulites deform affinely at least up to $60-\%$ elongation. It was also indicated that the spherulite deformation model of the multiple concentric-shell deformation is not appropriate for describing the observed ring scattering patterns.

KEY WORDS Ring Scattering / Helicoidal Twisting of Lamellae / Spherulite Deformation / Polyethylene / Affine Deformation /
\end{abstract}

Light scattering technique has been applied for investigating the spherulitic crystalline superstructure and its deformation mechanism. The size of the spherulites and the change in shape upon sample deformation have been analyzed from the small-angle light scattering patterns.

For the banded spherulite in which lamellae coherently twist in the spherulite with a periodic distance $d$, the scattering maximum (so-called "ring" scattering) has been observed and theoretically predicted at the scattering angle reciprocally related to a half of the periodic distances,

$$
2 \rho \sin \left(\theta_{\max } / 2\right)=\lambda
$$

where $\rho=d / 2, \lambda$ is the wavelength of light in the medium, and $\theta_{\max }$ is the scattering angle at which the ring scattering passes maximum intensity. The ring scattering also gives direct information on spherulite deformation mechanism.

* Present Address: The Research Center, Mitsui Petrochemical Industries, Ltd., Waki-cho, Kuga-gun, Yamaguchi 740, Japan.
Variation of the ring scattering with time under stress relaxation and creep experiments is anticipated to give direct information on the relaxation associated with the lamellar twisting or detwisting process. The deformation process involving the lamellar twisting and detwisting has recently been proposed as one of the structural origins of $\alpha_{1}$ crystalline dispersion (i.e., the lower temperature $\alpha$ mechanical dispersion associated with crystalline region) from rheo-optical studies of the films crystallized from oriented melts. ${ }^{1,2}$ Thus, the studies of ring scattering would be of importance in understanding the nature of the $\alpha$-mechanical dispersion of crystalline polymers. The deformation process of the ring structure and its time dependence can be studied as a function of the angle that the radial fibrils of the spherulites make with respect to the stretching direction. Thus the anisotropy of the structural response can be studied, in principle, which is one of the strongest merits in studying the ring scattering as a rheo-optical study. 
In this article we will study the effects of the optical axis orientation (i.e., tilting and twisting of the optical axes with respect to spherulite radii) and polarization conditions $\left(H_{\mathrm{V}}, V_{\mathrm{V}}\right.$, and $\left.H_{\mathrm{H}}\right)$ on the scattering angle $\theta_{\max , \mu}$ where the ring scattering takes on maximum intensity at azimuthal angle $\mu$. We will also study the ring spacing as a function of the azimuthal angle between an angular portion of the spherulite under consideration and the stretching direction. These experimental results will be compared with those expected for the affine deformation.

\section{TEST SPECIMENTS AND EXPERIMENTAL PATTERNS}

The light scattering patterns were made on a medium density polyethylene (Sumikasen, Sumitomo Chemical Co., Ltd., Japan). Gel-permeation chromatography indicates a number-average molecular weight of 19,300 , and a weight-average molecular weight of 170,000 . The degree of branching is $1.3 \mathrm{CH}_{3} / 100 \mathrm{C}$ (measured from infrared absorbances in the melt at 1376 and 1368 $\mathrm{cm}^{-1}$ ). Pellets were melted at $160^{\circ} \mathrm{C}$ for about $10 \mathrm{~min}$ in a laboratory press and pressed to a film of 100-micron thickness between sheets of mylar. The films were then allowed to cool to room temperature at a natural rate of cooling of the press (about $6 \mathrm{~h}$ ). The density measured by the density gradient tecnique in a water-methanol mixture is $0.937 \mathrm{~g} / \mathrm{cm}^{3}$. The weight average crystallinity is about $60 \%$, as measured by density, X-ray, and differential scanning calorimetry at room temperature. The sample is identical with that studied by Hashimoto, et al., on dynamic light scattering. ${ }^{3,4}$

Figure 1 represents variations of $H_{\mathrm{V}}, V_{\mathrm{v}}$, and $H_{\mathrm{B}}$ small-angle light scattering patterns with elongation percents (the stretching direction is vertical). The polarization direction of the incident light wave is vertical for the $H_{\nabla}$ and $V_{\mathrm{v}}$ patterns, and horizontal for the $H_{\mathrm{B}}$ patterns, while the polarization direction of the analyzer set between the specimen and photographic film is vertical for the $V_{\mathrm{V}}$ pattern and horizontal for the $H_{\mathrm{V}}$ and $H_{\mathrm{H}}$ patterns.

The $H_{\mathrm{v}}$ pattern for the undeformed specimen has a maximum intensity at a scattering angle $\theta_{\max }=$ $1.67^{\circ}$ within the specimen as shown in Figure 3 (corresponding to the scattering angle in air $2.49^{\circ}$, assuming refractive index of the specimen 1.5). By applying the Stein-Rhodes' equation, ${ }^{14}$

$$
U_{\max }=4 \pi(R / \lambda) \sin \left(\theta_{\max } / 2\right)=4.1
$$

where $R$ is the average spherulite radius, the average diameter of the spherulites was found to be 19 microns.

The $H_{\nabla}$ pattern becomes elliptical with the long axis oriented on the equator with elongation of the specimens along a vertical direction, indicating that the spherulites are deformed to an ellipsoidal shape with the long axis parallel to the elongation direction. With increasing elongations, the intensity at the center of the pattern, and along meridian $\left(\mu=0^{\circ}\right)$ and equator $\left(\mu=90^{\circ}\right)$ increases owing to increasing internal disorders of the spherulites with deformation. $^{5-8}$

The $H_{\mathrm{v}}$ pattern depends only upon the orientation and anisotropy fluctuations, while the $V_{\mathbf{v}}$ and $H_{\mathrm{H}}$ patterns depend upon the density fluctuations as well. The relative contribution of the two types of fluctuations may be studied by comparing the $H_{\mathrm{H}}$ and $V_{\mathrm{v}}$ patterns. If the density fluctuations are predominant over the orientation and anisotropy fluctuations, the $H_{\mathrm{H}}$ and $V_{\mathrm{v}}$ patterns should be identical. The $H_{\mathrm{H}}$ and $V_{\mathrm{v}}$ patterns in Figure 1 are different especially at the low elongation percents, indicating that the orientation and anisotropic fluctuations contribute to scattering predominantly over the other. However with increasing elongation, the density contribution becomes increasingly significant, so that the difference between the $H_{\mathrm{H}}$ and $V_{\mathrm{V}}$ patterns become less.

Figure 2 represents the wide-angle ring scattering arising from the ring structure. The ring scattering intensity is generally very weak compared with the intensity of the small-angle scattering (order of $1 / 500$ of the small-angle scattering intensity). Therefore, the small-angle light scattering patterns as shown in Figure 1 were overexposed in Figure 2 resulting in degenerated circular patterns. Maximum ring scattering intensity for the undeformed specimens was observed at $\theta_{\max }=$ $18.2^{\circ}$ within the specimen (corresponding to the scattering angle in air $28.5^{\circ}$, assuming refractive index of the specimen 1.5 ;). By applying eq 1 , the ring spacing $\rho$ is 1.3 microns.

The change of the ring scattering with elongation is, in general, identical to that reported by Moore, et al. ${ }^{9,10}$ With increasing elongation, (i) 


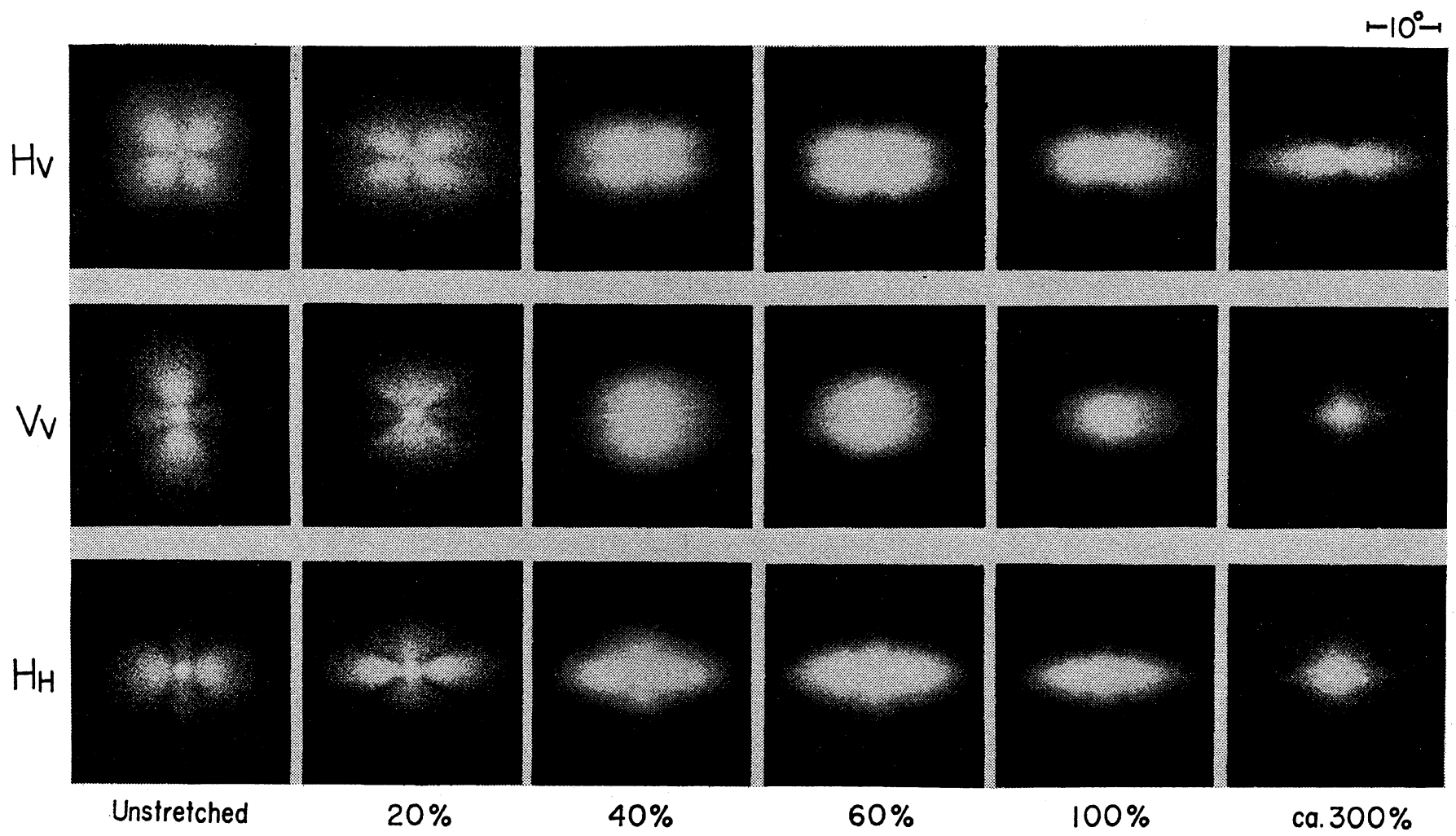

Figure 1. Variations of $H_{\mathrm{V}}, V_{\mathrm{V}}$ and $H_{\mathrm{H}}$ small-angle light scattering patterns with elongation percents. Stretching direction is in vertical. 


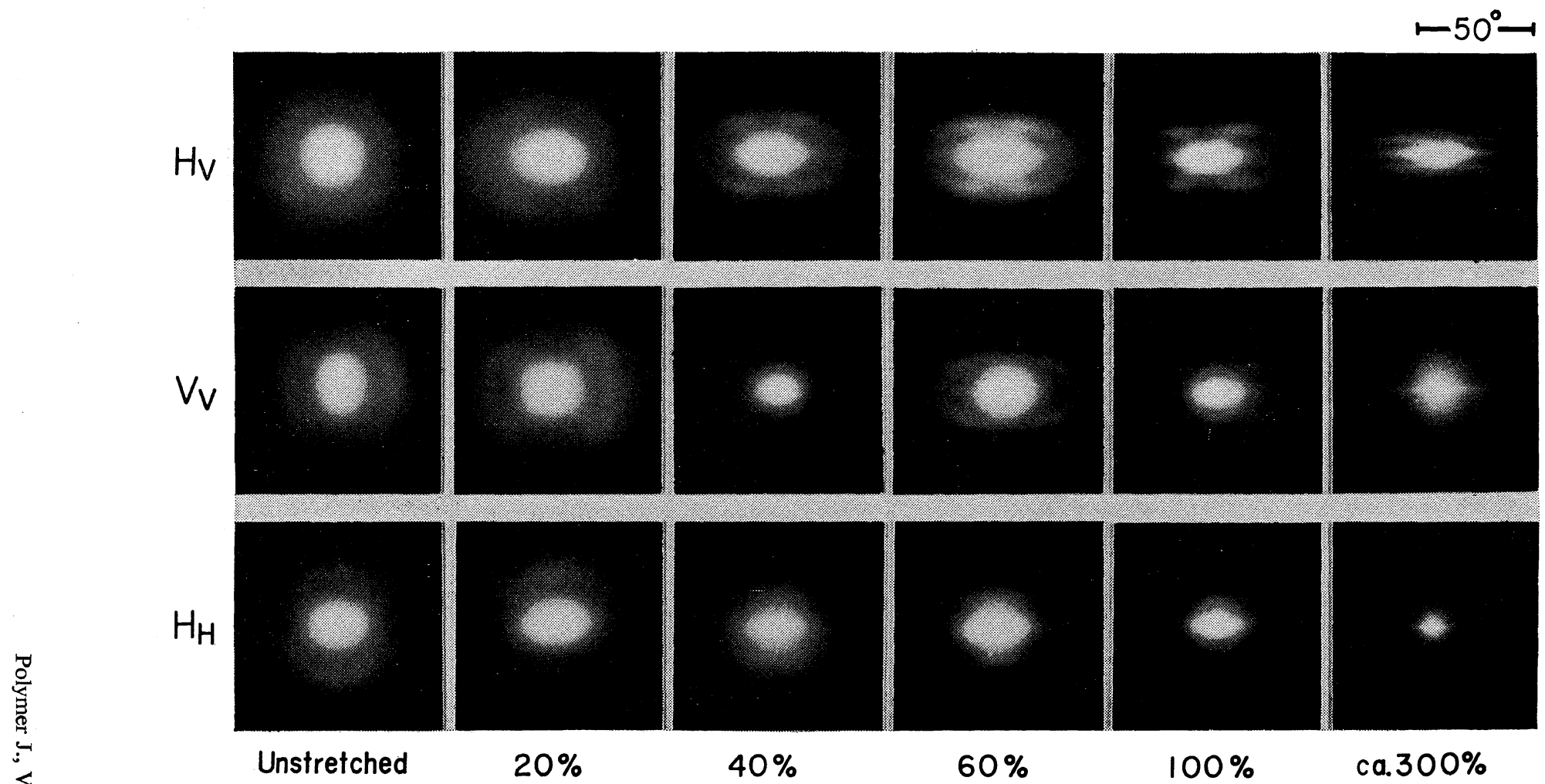

Figure 2. Variations of $H_{\mathrm{v}}, V_{\mathrm{v}}$, and $H_{\mathrm{H}}$ ring scattering patterns with elongation percents. Stretching direction is vertical. 
the ring spacing along the stretching direction increases (as seen by the fact that the $H_{\mathrm{H}}$ ring scattering shifts toward small scattering angle), while the spacing perpendicular to the stretching direction decreases (as seen by the fact that the $V_{\mathrm{V}}$ scattering shifts toward large scattering angle). Therefore, the ring structure also becomes ellipsoidal with the major axis orients toward the stretching direction accompanied by spherulite deformation. (ii) The equatorial arcs of the $V_{v}$ ring scattering split into four arcs owing to the increasing disorder of the optical axis orientation and a decrease in the ring fraction of the lamellae coherently twisted to form a ring structure in the equatorial part of the spherulites (to be described in detail later). Also (iii) the $H_{\mathrm{H}}$ scattering becomes less intense with increasing elongation owing to the chain tilting process in the meridional part of the spherulite (i.e., that part of the spherulites parallel to the stretching direction). Moreover, it is found as in the Moore's work that the $H_{\mathrm{V}}$ scattering arcs and the splitted $V_{\mathrm{v}}$ arcs tend to be tilted so that they are nearly parallel to the equator and are simultaneously shifted slightly toward the meridian with increasing elongation $(i . e$. , the azimuthal angle $\mu_{\max }$ at which the scattering becomes maximum tends to decrease slightly). These aspects of the ring scattering will be described in later.

Although Moore reported the deformation of the ringed spherulites of high-density polyethylene at relatively low elongation percents (up to about $30 \%$ ), we were able to observe it up to very high elongations. For the particular test specimen studied, the ring structure was conserved even at about $300-\%$ elongation probably in the polar parts of fairly disintegrated spherulites. The elongation ratio of the ring spacing is still roughly identical to that of the bulk specimen.

In the next section, we shall discuss the effects of tilting and twisting of the optical axis and the ring fraction on ring scattering, and especially these effects on the ring spacing as estimated from the scattering angle $\theta_{\max }$ at which the scattering becomes maximum. We shall then investigate the angular dependence of the ring spacing as a function of elongation percents in connection with the spherulite deformation mechanism.

\section{MODEL AND THEORETICAL CALCULATIONS}

The scattering of light by deformed ringed spherulites was first calculated by van Aartsen and Stein ${ }^{11}$ for a limited number of cases based upon the affine deformation of two-dimensional spherulite. We will restrict our treatments also to twodimensional spherulites for the sake of simplicity in numerical calculations. Though the two-dimensional model is less appropriate than the three-dimensional spherulite models, the two-dimensional model will suffice in our studies of establishing qualitatively the effects of the optical axis orientation on the scattering angle of the maximum ring scattering.

The model employed in this article and the principle of the calculations are identical to those employed in the studies by van Aartsen and Stein. The calculations for the two-dimensional ringed spherulites are generalized, as in the calculations of the three-dimensional nonringed spherulites, ${ }^{12,13}$ to include the scattered intensity for parallel and crossed polarizers, $I_{/ /}(\psi)$ and $I_{+}(\psi)$, where $\psi$ is the angle between polarization direction of the incident beam and the vertical direction. As in the earlier works $H_{\mathrm{v}}, V_{\mathrm{H}}, H_{\mathrm{H}}$, and $V_{\mathrm{v}}$ scattering correspond to $I_{+}\left(\psi=0^{\circ}\right), I_{+}\left(\psi=90^{\circ}\right), I_{/ /}\left(\psi=90^{\circ}\right)$, and $I_{I /}\left(\psi=0^{\circ}\right)$, respectively. The general equations for the 0 vectors (the unit vectors parallel to the polarization direction of analyzer) are given by eq 32 and 33 in the article of van Aartsen and $\operatorname{Stein}^{13}$ to permit rigorous calculations of the scattered intensity at the large scattering angles.

The calculations were performed both for the model I and II of the affine deformation of the spherulites ${ }^{11-13}$ but the results presented here are only for the model I, since the results for model II are almost identical to those for the model I, and the model I is physically more reasonable than the model II. For model I, the number of scattering elements per unit area remains constant during deformation whereas with model II, the number of elements per unit angle is held constant.

Reorientation of the optical axis with respect to the spherulite radii was introduced into the ringedspherulite deformation. The tilting and rotation angle of the optical axis of the type as described by van Aartsen and Stein ${ }^{12,13}$ for nonringed spherulites were introduced into the ringed spherulites, 


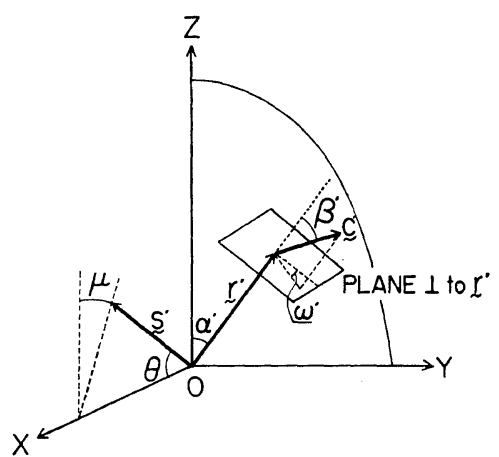

Figure 3. Definitions of the scattering angle $\theta$ and azimuthal angle $\mu$ and of the orientation of the principal optical axis (unit vector $\mathbf{c}^{\prime}$ ) with respect to spherulite radius $\mathbf{r}^{\prime}$ for the deformed two-dimensional spherulite. The angle $\beta^{\prime}$ and $\omega^{\prime}$ are the polar and azimuthal angles of the optical axis with respect to $\mathbf{r}^{\prime}$. $\mathbf{s}^{\prime}$ is a unit vector parallel to the propagation direction of the scattered beam.

$$
\begin{gathered}
\text { tilting; } \beta^{\prime}=\beta_{0} \exp \left[-\kappa\left(\lambda_{3}{ }^{2}-\lambda_{2}{ }^{2}\right) \cos ^{2} \alpha^{\prime}\right] \\
\text { rotation; } g=2\left\langle\cos ^{2} \omega^{\prime}\right\rangle_{\mathrm{av}}-1 \\
=1-\exp \left[-\eta\left(\lambda_{3}{ }^{2}-\lambda_{2}{ }^{2}\right) \sin ^{2} \alpha^{\prime}\right]
\end{gathered}
$$

where $\beta^{\prime}$ and $\omega^{\prime}$ are the polar and azimuthal angles of the optical axis (unit vector $\mathbf{c}^{\prime}$ ) with respect to the spherulite radii (see Figure 3), and $\kappa$ and $\eta$ are the parameters related to ease of the optical axis reorientation. $\lambda_{3}$ and $\lambda_{2}$ are the extension ratios parallel and perpendicular to the stretching direction, respectively.

The rotation of the optical axis around the spherulite radii is in part associated with the $c$-axis orientation mechanism of crystallites as visualized by the rotation of the $c$ - and $a$-axis around the spherulite radii (crystal $b$-axis). If all fractions of the lamellae twist helicoidally in a manner as specified by the linear increase of $\omega^{\prime}$ with distance $r^{\prime}$ from the center,

$$
\omega^{\prime}\left(r^{\prime}\right)=\left(2 \pi n / R^{\prime}\right) r^{\prime}
$$

there would be no such $c$-axis orientation mechanism, and therefore such model is incapable of describing the observed crystal orientation. In eq $5, n$ is the number of complete turns of the optic axis with the radial fibrils of the spherulite of radius $R^{\prime}$ and located at the angular position $\alpha^{\prime}$.

The $c$-axis orientation mechanism of the ringed spherulite may be envisaged only in terms of non- linear increase of $\omega^{\prime}$ with $r^{\prime}$ and/or of decreasing fraction $f_{\mathrm{r}}$ of the lamellae involved in helicoidal twisting. In this article, we treat the latter case, the deformation mechanism of which was newly introduced in the model calculation. Therefore, the term like $\cos ^{2} \omega^{\prime}$ is given by a weighted average of the helicoidally twisted lamellae, $\cos ^{2} \omega^{\prime}\left(r^{\prime}\right)$, (for which $\omega^{\prime}$ is given by eq 5 and of the nonringed lamellae, $\left\langle\cos ^{2} \omega^{\prime}\right\rangle_{\mathrm{av}}$;

$$
\cos ^{2} \omega^{\prime}=f_{\mathrm{r}} \cos ^{2} \omega^{\prime}\left(r^{\prime}\right)+\left(1-f_{\mathrm{r}}\right)\left\langle\cos ^{2} \omega^{\prime}\right\rangle_{\mathrm{av}}
$$

The average value $\left\langle\cos ^{2} \omega^{\prime}\right\rangle_{\mathrm{ar}}$ was assumed to be given by eq 4, and thus is a function of $\alpha^{\prime}$ and of the extension ratios $\lambda_{3}$ and $\lambda_{2}$ parallel and perpendicular to the stretching direction, respectively. The fraction $f_{r}$ is also a function of $\alpha^{\prime}$, in general, which is given by an empirical function,

$$
f_{\mathbf{r}}=f_{\mathbf{r}}^{0} \exp \left[-f\left(\lambda_{3}{ }^{2}-\lambda_{2}{ }^{2}\right) \sin ^{2} \alpha^{\prime}\right]
$$

The final formulae of the scattered electric fields under the crossed $\left(E_{+}\right)$and parallel polarizers $\left(E_{\| /}\right)$ are given by

$$
\begin{aligned}
& \left(\begin{array}{l}
E_{+} \\
E_{/ /}
\end{array}\right)=\text {(const.) }\left(\frac{R}{W^{*}}\right)^{2}\left\{-\left(\begin{array}{l}
b_{+} \\
b_{\| /}
\end{array}\right) E_{1} \sin \psi-\right. \\
& {\left[\left(\begin{array}{l}
b_{+} \\
b_{\| /}
\end{array}\right) \cos \psi-\left(\begin{array}{l}
c_{+} \\
c_{\| /}
\end{array}\right) \sin \psi\right] E_{2}+\left(\begin{array}{l}
c_{+} \\
c_{\| /}
\end{array}\right) E_{3} \cos \psi} \\
& \left.+\left[\left(\begin{array}{l}
c_{+} \\
c_{\| /}
\end{array}\right) \cos \psi-\left(\begin{array}{l}
b_{+} \\
b_{\| /}
\end{array}\right) \sin \psi\right] E_{4}\right\}
\end{aligned}
$$

where

$$
\begin{aligned}
& b_{+}=Q_{+}\left[\sin ^{2} \theta \cos \mu \sin (\mu+\psi)-\cos \psi\right] \\
& c_{+}=Q_{+}\left[\sin ^{2} \theta \cos \mu \sin (\mu+\psi)-\sin \psi\right] \\
& Q_{+}=-\left[1-\sin ^{2} \theta \sin ^{2}(\mu+\psi)\right]^{-1 / 2} \\
& b_{/ /}=Q_{/ /}\left[\sin ^{2} \theta \sin \mu \cos (\mu+\psi)+\sin \psi\right] \\
& c_{/ /}=Q_{\| /}\left[\sin ^{2} \theta \cos \mu \cos (\mu+\psi)-\cos \psi\right] \\
& Q_{l /}=-\left[1-\sin ^{2} \theta \cos ^{2}(\mu+\phi)\right]^{-1 / 2}
\end{aligned}
$$

and

$$
W^{*}=(2 \pi / \lambda) R \sin \theta\left[\lambda_{2}{ }^{2} \sin ^{2} \mu+\lambda_{3}{ }^{2} \cos ^{2} \mu\right]^{1 / 2}
$$

The angle $\theta$ and $\mu$ are the scattering and azimuthal angles, respectively, $\lambda$ is the wavelength of light in the medium, and $R$ is the radius of undeformed spherulite.

$$
\begin{aligned}
E_{1}= & \int_{\alpha=0}^{2 \pi} \int_{q=0}^{W *}\left[-\left(\cos ^{2} \beta^{\prime}-\sin ^{2} \beta^{\prime} \cos ^{2} \omega^{\prime}\right) \cos ^{2} \alpha^{\prime}\right. \\
& \left.+\cos ^{2} \beta^{\prime}\right] \times \cos [q \cos (\alpha-\gamma)] \mathrm{d} \alpha q \mathrm{~d} q
\end{aligned}
$$




$$
\begin{aligned}
E_{2}= & \int_{\alpha=0}^{2 \pi} \int_{q=0}^{W *}\left(\cos ^{2} \beta^{\prime}-\sin ^{2} \beta^{\prime} \cos ^{2} \omega^{\prime}\right) \sin \alpha^{\prime} \cos \alpha^{\prime} \\
& \times \cos [q \cos (\alpha-\gamma)] \mathrm{d} \alpha q \mathrm{~d} q \\
E_{3}= & \int_{\alpha=0}^{2 \pi} \int_{q=0}^{W *}\left[-\left(\cos ^{2} \beta^{\prime}-\sin ^{2} \beta^{\prime} \cos ^{2} \omega^{\prime}\right) \sin ^{2} \alpha^{\prime}\right. \\
& \left.+\cos ^{2} \beta^{\prime}\right] \times \cos [q \cos (\alpha-\gamma)] \mathrm{d} \alpha q \mathrm{~d} q \\
E_{4}= & p \int_{\alpha=0}^{2 \pi} \int_{q=0}^{W *} \cos [q \cos (\alpha-\gamma)] \mathrm{d} \alpha q \mathrm{~d} q \\
= & 2 \pi p W^{*} J_{1}\left(W^{*}\right)
\end{aligned}
$$

where $\gamma$ and $\alpha$ are satisfying

$$
\begin{aligned}
& \sin \gamma=\lambda_{2}\left[\lambda_{2}{ }^{2} \sin ^{2} \mu+\lambda_{3}{ }^{2} \cos ^{2} \mu\right]^{-1 / 2} \sin \mu \\
& \cos \gamma=\lambda_{3}\left[\lambda_{2}{ }^{2} \sin ^{2} \mu+\lambda_{3}{ }^{2} \cos ^{2} \mu\right]^{-1 / 2} \cos \mu \\
& \sin \alpha^{\prime}=\lambda_{2}\left[\lambda_{2}{ }^{2} \sin ^{2} \alpha+\lambda_{3}{ }^{2} \cos ^{2} \alpha\right]^{-1 / 2} \sin \alpha \\
& \cos \alpha^{\prime}=\lambda_{3}\left[\lambda_{2}{ }^{2} \sin ^{2} \alpha+\lambda_{3}{ }^{2} \cos ^{2} \alpha\right]^{-1 / 2} \cos \alpha
\end{aligned}
$$

The parameter $p$ characterizes a relative contribution of the density fluctuations $\left(\alpha_{2}-\alpha_{\mathrm{s}}\right)$, and the anisotropy fluctuations $\left(\alpha_{1}-\alpha_{2}\right)$, and is defined by

$$
p=\left(\alpha_{2}-\alpha_{\mathrm{s}}\right) /\left(\alpha_{1}-\alpha_{2}\right)
$$

where $\alpha_{1}$ and $\alpha_{2}$ are the polarizabilities of the scattering element parallel and perpendicular to the optical axis, respectively, and $\alpha_{\mathrm{s}}$ is the polarizability of the surrounding medium assumed to be isotropic.

The angle $\beta^{\prime}$ is given by eq 3 where $\beta_{0}$ and $\kappa$ are the parameters to be assigned and $\cos ^{2} \omega^{\prime}$ is given by eq 4 to 6 . It should be noted that the following terms appearing in eq 8 vanish owing to a symmetry requirement of $\omega^{\prime}$.

$$
\begin{gathered}
I_{1}=\int_{\alpha=0}^{2 \pi} \int_{q=0}^{W *} \sin 2 \beta^{\prime} \sin \omega^{\prime} \sin \alpha^{\prime} \\
\quad \times \cos [q \cos (\alpha-\gamma)] \mathrm{d} \alpha q \mathrm{~d} q \\
I_{2}=\int_{\alpha=0}^{2 \pi} \int_{q=0}^{W *} \sin ^{2} \beta^{\prime} \sin 2 \omega^{\prime} \cos \alpha^{\prime} \\
\quad \times \cos [q \cos (\alpha-\gamma)] \mathrm{d} \alpha q \mathrm{~d} q \\
I_{3}=\int_{\alpha=0}^{2 \pi} \int_{q=0}^{W *} \sin 2 \beta^{\prime} \cos \omega^{\prime} \sin 2 \alpha^{\prime} \\
\times \cos [q \cos (\alpha-\gamma)] \mathrm{d} \alpha q \mathrm{~d} q \\
I_{4}=\int_{\alpha=0}^{2 \pi} \int_{q=0}^{W *} \sin 2 \beta^{\prime} \cos \omega^{\prime} \cos 2 \alpha^{\prime} \\
\times \cos [q \cos (\alpha-\gamma)] \mathrm{d} \alpha q \mathrm{~d} q
\end{gathered}
$$

The symmetry requirement arises from the physi- cal indistinguishability of the optical axis orientations designated by the unit vectors $\mathbf{c}^{\prime}$ and $-\mathbf{c}^{\prime}$.

According to the affine deformation, it immediately follows that

$$
R^{\prime}=R\left[\lambda_{2}{ }^{2} \sin ^{2} \alpha+\lambda_{3}{ }^{2} \cos ^{2} \alpha\right]^{1 / 2}
$$

Consequently, the ring spacings for the undeformed and deformed spherulites ( $\rho^{\prime}$ and $\rho$, respectively) are given by

$$
\rho^{\prime}=\rho\left[\lambda_{2}{ }^{2} \sin ^{2} \alpha+\lambda_{3}{ }^{2} \cos ^{2} \alpha\right]^{1 / 2}
$$

\section{CALCULATED RING SCATTERING AND THE EFFECT OF OPTICAL AXIS ORIENTATION}

The calculated scattering patterns were ploted with the reduced scattering angle $W=2 \pi(R / \lambda)$ $\sin \theta$ in radial direction and with the azimuthal angle $\mu$ in tangential direction. The $V_{\mathrm{V}}$ and $H_{\mathrm{H}}$ patterns depends on a parameter $p=\left(\alpha_{2}-\alpha_{\mathrm{s}}\right) /$ $\left(\alpha_{1}-\alpha_{2}\right)$, which is assigned to be $-1 / 2$. In order to study ring scattering as a function of the elongation ratio $\lambda_{B}$ and internal orientation of the optical axis, the ring spacing $\rho$ of the undeformed spherulite was set equal to $\rho / R=1 / 8$. The angle $\beta_{0}$ was always set equal to 90 degrees.

Figures 4 and 5 represent the calculated smallangle light scattering patterns and wide-angle ring scattering, respectively, under the $H_{\mathrm{V}}, V_{\mathrm{V}}$, and $H_{\mathrm{H}}$ polarizations for the two-dimensional spherulite uniaxially stretched by $\lambda_{\mathrm{B}}=\lambda_{3}=1.2$ under constant volume $\lambda_{3} \lambda_{2}=1$ (the stretching direction is in vertical).

The patterns (a) to (d) in these figures represent the effect of orientation of the optical axis within the deformed spherulites, and were obtained for (a) $\kappa=\eta=0, f_{\mathrm{r}}=1$, (b) $\kappa=0.5, \eta=0, f_{\mathrm{r}}=1$, (c) $\kappa=0$, $\eta=1, f_{\mathrm{r}}^{0}=0.5, f=0$, and (d) $\kappa=0.5, \eta=1, f_{\mathrm{r}}^{0}=0.5$ and $f=0$. Thus the patterns in (a) and (b) show the effect of the tilting of the optical axis (for positive values of $\kappa$, the tilting being dominant in the polar parts of the deformed spherulites), while those in (a) and (c) show the effect of the rotation of the optical axis (for positive values of $\eta$, the rotation being dominant in the equatorial parts of the spherulites). The patterns in (a) and (d) then represent a combined effect of the tilting and rotation.

The effects of the internal orientation of the 

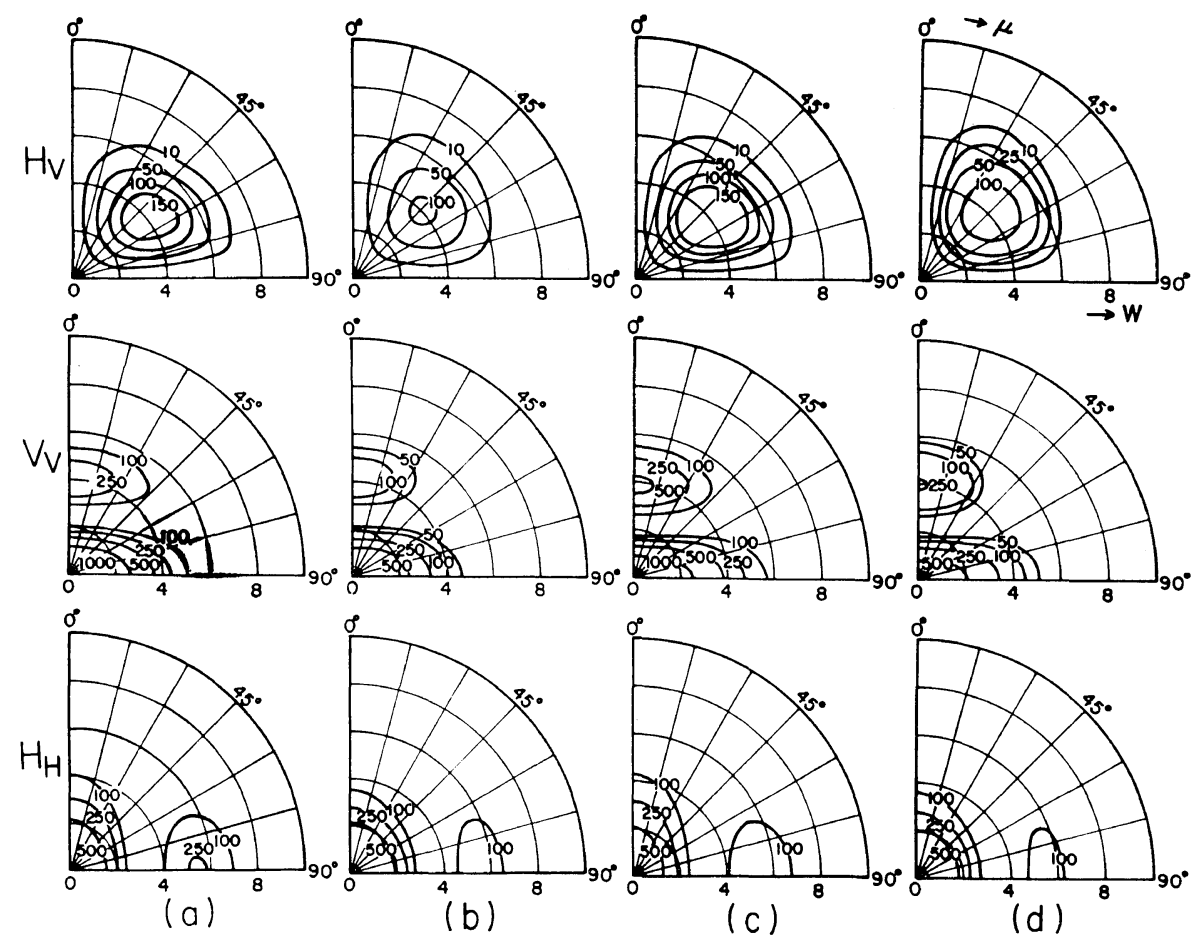

Figure 4. Calculated $H_{\mathrm{V}}, V_{\mathrm{V}}$, and $H_{\mathrm{H}}$ small-angle light scattering patterns for the spherulite uniaxially stretched by $\lambda_{\mathrm{B}}=\lambda_{3}=1.2$ under constant volume $\lambda_{3} \lambda_{2}=1$. Stretching direction is in vertical: (a) $\kappa=\eta=0$ and $f_{\mathrm{r}}=1$; (b) $\kappa=0.5, \eta=0$ and $f_{\mathrm{r}}=1$; (c) $\kappa=0, \eta=1, f_{\mathrm{r}}{ }^{0}=0.5$, and $f=0$; (d) $\kappa=0.5, \eta=1, f_{\mathrm{r}}^{\circ}=0.5$, and $f=0$.

optical axis on the spherulitic scattering (Figure 4) turned out to be essentially identical to those obtained by van Aartsen and Stein for the nonringed three-dimensional spherulites. Since we are primarily interested in studying the effects on the ring scattering, we will not further discuss the smallangle scattering.

The calculated ring scattering patterns (Figure 5) are in agreement with the experimental patterns; the $H_{\mathrm{H}}$ and $V_{\mathrm{V}}$ scattering arcs shift toward smaller and larger angles, and the $H_{\mathrm{v}}$ patterns become elliptical in shape and oriented along the equator. The reduced scattering angle $W_{\max , \mu}$ at which the scattering at a given azimuthal angle $\mu$ passes through a maximum value turns out to be independent of the parameters, $\kappa, \eta$ and $f_{r}$, i.e., the orientation of the optical axis within the spherulites. Rather, the internal reorientation processes related to these parameters affect only the angular dependence of the maximum intensity (the intensity at $\left.W_{\max , \mu}\right)$ with respect to $\mu$ and the ab- solute intensity level.

Figure 6 shows one quarter of the contour plots of the $W_{\max }, \mu$ values for the $H_{\mathrm{v}}$ (in the second quadrant), $H_{\mathrm{B}}$ (in the third quadrant) and $V_{\mathrm{V}}$ (in the first and fourth quadrants) ring scattering calculated for $\lambda_{\mathrm{B}}=1.2$. These contour lines obtained under different polarization conditions can be superposed into a single contour line (as shown in the contour line in the first quadrant marked "total"), indicating that the peak position of the ring scattering $W_{\max , \mu}$ is also independent of the polarization conditions but dependent only upon the angular dependence of the ring scattering $\rho^{\prime}$ with respect to $\alpha$. The effect of the internal orientation of the optical axes on the $H_{\mathrm{v}}$ scattering, for an example, is most clearly observed in the change of the intensity distribution along the contour line as demonstrated in the change of the positions (a) to (d) of the absolute intensity maximum, (the points (a) to (d) correspond to the position of the intensity maximum of Figures $5(\mathrm{a})$ to $5(\mathrm{~d})$, 

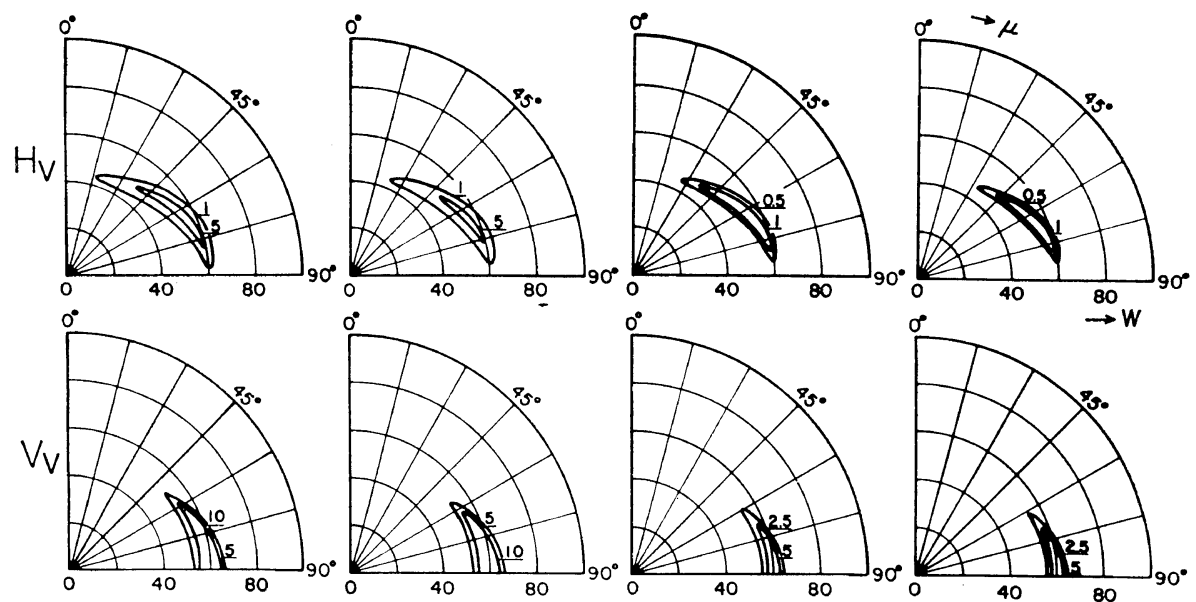

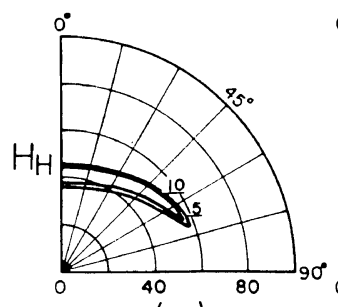

(a)

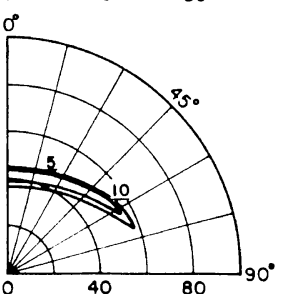

(b)

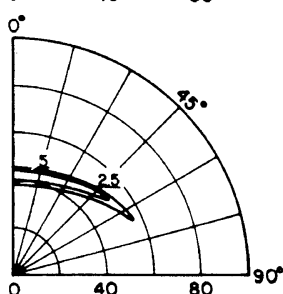

(c)
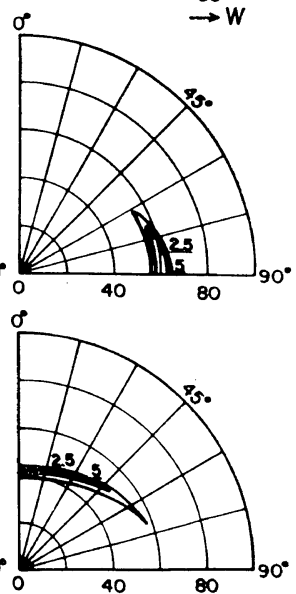

(d)

Figure 5. Calculated $H_{\mathrm{V}}, V_{\mathrm{V}}$, and $H_{\mathrm{H}}$ light scattering patterns for the deformed spherulite as in Figure 4. The patterns (a) to (d) are obtained by using the same parameters as in Figure 4.

respectively).

It was found that the position $W_{\max , \mu}$ can be predicted from the ring spacing $\rho^{\prime}(\alpha)$ at an position corresponding to $\alpha=\mu$ of the deformed spherulite by using Bragg's law, i.e.,

$$
W_{\max , \mu}=2 \pi(R / \lambda) \sin \theta_{\max , \mu=\alpha}
$$

and $\theta_{\max , \mu=\alpha}$ is given by

$$
2 \rho^{\prime}(\alpha) \sin \left(\theta_{\max , \mu=\alpha} / 2\right)=\lambda
$$

where $\rho^{\prime}(\alpha)$ is given by eq 26 . It should be noted that the ring scattering at $\mu=\alpha$ is reciprocally related to the ring spacing at $\alpha$ of the spherulite. The $W_{\max , \mu}$ determined by Bragg's equation, is also included in Figure 6 (solid circles). The equation found applicable, at least, up to $60-\%$ elongation, can thus provide a method to evaluate the angular dependence of the ring spacing for deformed spherulites as will be described in detail next section.

Figure 7 represents the effect of increasing the spherulite deformation $\left(\lambda_{B}=1.4\right)$ on the $H_{v}, V_{v}$, $H_{\mathrm{H}}$ ring scattering patterns for the case of $\kappa=\eta=0$ and $f_{\mathrm{r}}=1$, i.e., the case corresponding to Figure

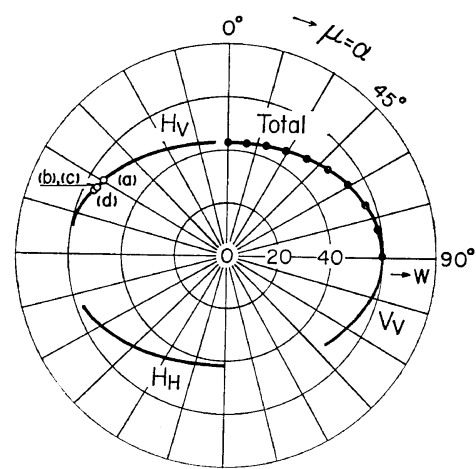

Figure 6. Contour plots of the reduced scattering angle $W_{\max , \mu}=4 \pi(R / \lambda) \sin \left(\theta_{\max , \mu} / 2\right)$ of the maximum ring scattering at particular $\mu$ for the $H_{\mathrm{V}}$ (in the second quadrant), $H_{\mathrm{H}}$ (in the third quadrant) and $V_{\mathrm{V}}$ (in the first and fourth quadrant) polarizations calculated for $\lambda_{B}=1.2$. Each contour line is superposable onto a single contour line as shown by the line (marked "total") in the first quadrant. The result shown by the solid circles is obtained by applying Bragg's law to the ring spacing $\rho^{\prime}(\alpha)$ at $\alpha$ and to the scattering angle $\theta_{\max , \mu=\alpha}$ of the maximum intensity at particular azimuthal angle $\mu=\alpha$. 


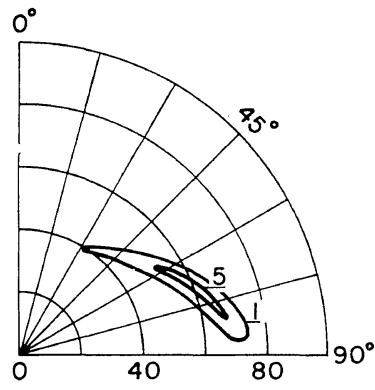

(a)

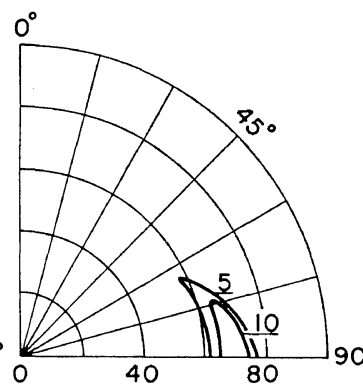

(b)

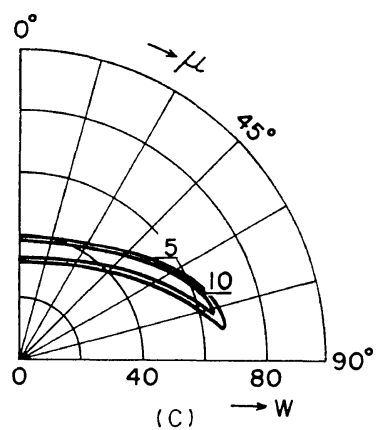

(C)

Figure 7. The calculated $H_{\mathrm{V}}$ (a), $V_{\mathrm{V}}(\mathrm{b})$, and $H_{\mathrm{H}}$ (c) ring scattering patterns for a higher elongation ratio $\lambda_{\mathrm{B}}=1.4$ and for $\kappa=\eta=0$ and $f_{\mathrm{r}}=1$, i.e., for the parameters corresponding to Figure 5(a).

5(a) for $\lambda_{\mathrm{B}}=1.2$. Upon increasing the elongation, the $V_{\mathrm{V}}$ and $H_{\mathrm{H}}$ scattering arcs shift more toward larger and smaller scattering angles, respectively, and the $H_{\mathrm{v}}$ pattern becomes an ellipse with a greater axial ratio, whose tendencies are in agreement with the experimental results. However, the calculated patterns deviate from the experimental patterns in that (i) the calculated $H_{\nabla}$ scattering maximum shifts toward equator, while the experimental $H_{\mathrm{v}}$ arcs tend to shift slightly toward the meridian, and that (ii) the calculated $V_{\mathrm{v}}$ pattern is incapable of describing the tendency of the splitting or the tendency of decreasing intensity at the equator as seen in Figure 2.

The discrepancies between the calculated and experimental patterns could be effectively solved by introducing the angular dependence of the ring fraction as formulated in eq 7 . Figure 8 represents this effect on the $H_{\mathrm{v}}$ and $V_{\mathrm{v}}$ scattering pat-

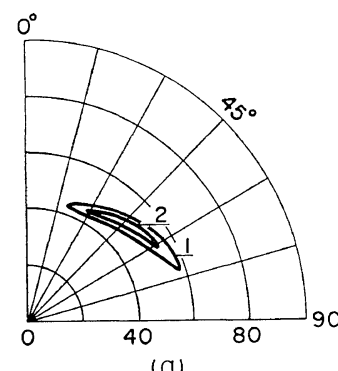

(a)

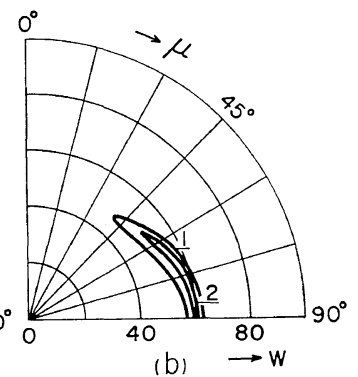

(b)
Figure 8. The calculated $H_{\mathrm{V}}$ (a) and $V_{\mathrm{V}}$ (b) ring scattering patterns for $\lambda_{B}=1.2$. The angular dependence of the ring fraction on the patterns was given by setting the parameters $f_{\mathrm{r}}{ }^{0}=1$ and $f=2, \kappa=$ $\eta=0$. terns for $\lambda_{\mathrm{B}}=1.2, \kappa=\eta=0$, and $f_{\mathrm{r}}^{0}=1$ and $f=2$. By comparing the results with those of Figure 5(a) the decreasing ring fraction in the equatorial part of the spherulites in fact decreases the discrepancies; it shifts the scattering maximum toward the meridian $\left(\mu_{\max }\right.$ being changed from $60^{\circ}$ for Figure 5(a) to $50^{\circ}$ for Figure 8(a)) and it strongly decreases the $V_{\mathrm{v}}$ intensity at the equator relative to other $\mu$ 's, leading to the tendency of splitted $V_{\mathrm{v}}$ arcs.

\section{SPHERULITE DEFORMATION}

The angular dependence of the spherulite deformation was studied by estimating and analyzing the angular dependence of the ring spacing based upon the method described in a previous section.

Figure 9 represents some typical results of the relationship between bulk elongation ratios $\lambda_{B}$ and elongation ratios of ring spacing $\lambda_{\mathrm{s}}$ in the polar $\left(\alpha=0^{\circ}\right)$, and equatorial $\left(\alpha=90^{\circ}\right)$ parts of the spherulites and in some intermediate parts $\left(\alpha=30^{\circ}\right.$ and $60^{\circ}$ ). The experimental values of $\lambda_{\mathrm{s}}$ were obtained as follows. The scattering angle $\theta_{\max , \mu=\alpha}$ at $\mu=\alpha$ was first measured from light scattering photographs. The spacing at $\alpha, \rho^{\prime}(\alpha)$ was estimated by using eq (27-b) and then $\lambda_{\mathrm{s}}$ at $\alpha$ was obtained from $\lambda_{\mathrm{s}}=\rho^{\prime}(\alpha) / \rho$. The solid lines were obtained by assuming affine deformation under constant volume with $\lambda_{\mathrm{B}}=\lambda_{3}$, and $\lambda_{1}=\lambda_{2}=\lambda_{\mathrm{B}}{ }^{-1 / 2}$, while the dash - dot lines were obtained for the same deformation with $\lambda_{\mathrm{B}}=\lambda_{3}=1 / \lambda_{2}$ in eq 26 .

The results shown in Figure 9 suggest that the spherulite deformation as observed from the deformation of the ring spacings obey the affine 

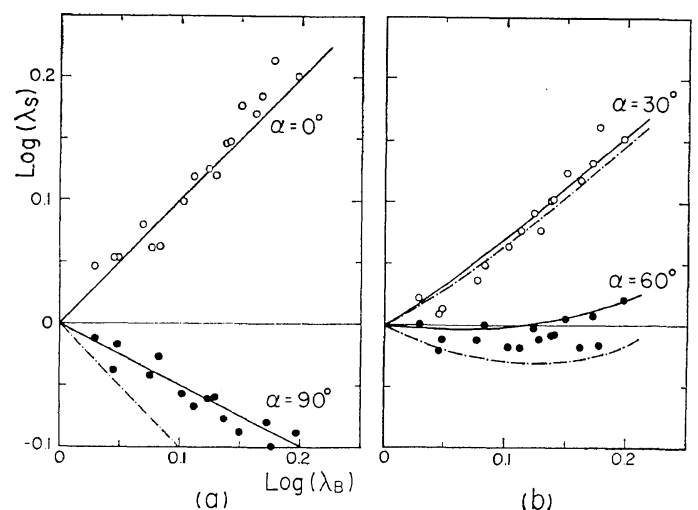

Figure 9. The relationship between the bulk elongation ratios $\lambda_{\mathrm{B}}$ and the elongation ratios of the ring spacing $\lambda_{\mathrm{s}}$ (a) in the polar $\left(\alpha=0^{\circ}\right)$ and in the equatorial parts $\left(\alpha=90^{\circ}\right)$ of the spherulites and (b) in some intermediate angular regions $\left(\alpha=30^{\circ}\right.$ and $\left.60^{\circ}\right)$. The solid and dash-dot lines were obtained by assuming affine deformation under constant volume with $\lambda_{\mathrm{B}}=\lambda_{3}, \lambda_{2}=\lambda_{1}=\lambda_{\mathrm{B}}{ }^{-1 / 2}$ (solid lines) and with $\lambda_{\mathrm{B}}=\lambda_{3}$, $\lambda_{2}=\lambda_{\mathrm{B}}{ }^{-1}, \lambda_{1}=1$ (dash-dot lines).

deformation under a constant volume up to a remarkably high elongation ratio $\left(\lambda_{\mathrm{B}} \cong 1.6\right)$. Obviously the affine deformation in three-dimensional space (i.e., $\lambda_{3}=\lambda_{\mathrm{B}}, \lambda_{2}=\lambda_{1}=\lambda_{\mathrm{B}}{ }^{-1 / 2}$ ) gives a better agreement with the experimental results for the three-dimensional spherulites than the affine deformation in two-dimensional space (i.e., $\lambda_{3}=\lambda_{B}$, $\lambda_{2}=\lambda_{\mathrm{B}}^{-1}, \lambda_{1}=1$ ).

\section{COMMENTS ON MULTIPLE CONCENTRIC-SHELL MODEL OF SPHERULITE DEFORMATION}

Wilchinsky ${ }^{15}$ first proposed the spherulite de- formation model of "multiple concentric-shell" deformation to explain the preferred orientation of the crystal $c$-axis in deformed polymers. According to this model, a spherical shell of the undeformed spherulite is deformed into an ellipsoidal shell of approximately uniform thickness within which the deforming strain tends to be equalized. In such a deformation, the local elongation ratios associated with the displacements of the crystals or scattering elements relative to each other are smaller in the outer shells and increase toward the inner shells.

There are some electron ${ }^{16}$ and optical microscopic ${ }^{17}$ evidence supporting or suggesting such deformation behavior of the spherulite, especially in the deformation of the spherulites of a more or less two-dimensional nature. The model has also been applied as a quantitative description of crystal orientation behavior of polyethylene spherulites accompanied by uniaxial stretching. ${ }^{18}$ Thus, it may be worthwhile to test the model in terms of the wide-angle light scattering technique.

The details of the ring scattering from deformed spherulites with multiple concentric-shells will be described elsewhere. ${ }^{19}$ In this section, we shall present only a typical set of calculated patterns (Figure 10). The numerical calculations were carried out for $\lambda_{B}=1.2$, the number of the shells 8 (in the interval between the neighbouring shells, the optical axis being rotated by $\pi$ radians) and for parameters, $\kappa=\eta=0$ and $f_{\mathrm{r}}=1$. The small-angle scattering patterns were almost identical to those shown in Figure 4. However, the ring scattering patterns shown in Figure 10 are remarkably different from those predicted by the affine deforma-

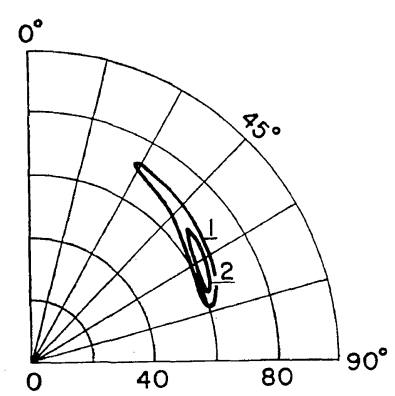

(a)

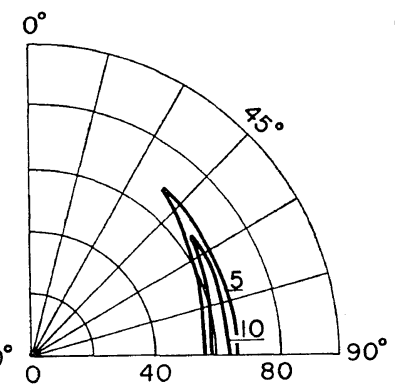

(b)

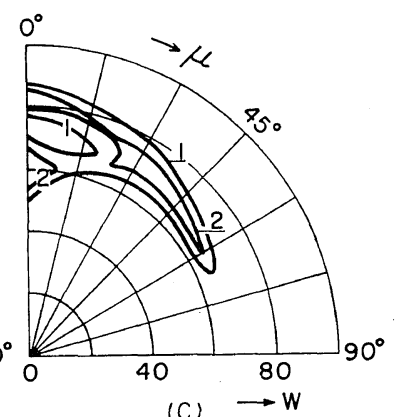

(C) $\rightarrow W$

Figure 10. The calculated $H_{\mathrm{V}}(\mathrm{a}), V_{\mathrm{\nabla}}(\mathrm{b})$, and $H_{\mathrm{H}}$ (c) ring scattering patterns for the spherulites with the multiple concentric-shell deformation $\lambda_{\mathrm{B}}=1.2, \kappa=\eta=0$, and $f_{\mathrm{r}}=1$. The number of shells is 8 . The optical axis is rotated by radians in the interval between the neighbouring shells. 


\section{A. Todo, T. Hashimoto, and H. KaWAI}

tion of the model I and also from the observed patterns. Thus the concentric-shell deformation model is incapable of describing the deformation of the three-dimensional spherulites in bulk. The affine deformation of model I and II seem more appropriate.

\section{CONCLUSION}

The deformation behavior of spherulites of a medium density polyethylene was studied by analyzing the change in the wide-angle ring scattering patterns arising from the helicoidal twisting of lamellae with applied uniaxial stretching. The ring scattering was calculated for two-dimensional ringed spherulites deformed affinely according to model I of van Aartsen and Stein ${ }^{11}$ as a function of elongation ratios, the internal reorientaion (tilting and twisting) of the optical axis, and of the fraction of ringed lamellae.

It turned out that the scattering angle $\theta_{\max , \mu}$ of the calculated ring scattering maximum at the azimuthal angle $\mu$ is independent of the polarization conditions and the manner of the internal orientation of the optical axis up to about $60-\%$ elongation, at least, and that $\theta_{\max , \mu}$ and the ring spacing of the spherulite at the angular region $\alpha=\mu$ of the spherulites with respect to the stretching direction are mutually related by Bragg's equation. An application of this rule to the spherulite deformation indicated that any angular portions of the spherulite deforms affinely up to high elongation percents (at least up to $60 \%$ ). It was also concluded that a better agreement between the calculated and observed patterns is obtainable by introducing an angular dependence into the fraction of the ringed lamellae in such a way that the ring fraction is minimum in the equatorial parts of the deformed spherulites and maximum in the polar parts.

Acknowledgements. This work was partly supported by a grant through the Japan-U.S. Cooperative Research Program, Japan Society for Promotion of Science and National Science Foundation, U.S.A., to which the authors are deeply indepted.
The authors are also indepted to the Mitsui Petrochemical Industries, Ltd., Tokyo, Japan, for financial support through a scientific grant.

\section{REFERENCES}

1. T. Hashimoto, N. Yasuda, S. Suehiro, S. Nomura, and H. Kawai, Polym. Prepr., Am. Chem. Soc., Div. Polym. Chem., 17, 118 (1976).

2. S. Suehiro, T. Yamada, T. Kyu, K. Fujita, T. Hashimoto, and H. Kawai, Polym. Eng. Sci., in press.

3. T. Hashimoto, R. E. Prud'homme, D. A. Keedy, and R. S. Stein, J. Polym. Sci., Polym. Phys. Ed., 11, 693 (1973).

4. T. Hashimoto, R. E. Prud'homme, and R. S. Stein, J. Polym. Sci., Polym. Phys. Ed., 11, 709 (1973).

5. R. S. Stein and W. Chu, J. Polym. Sci., Part A-a, 8, 1137 (1970).

6. T. Hashimoto and R. S. Stein, J. Ploym. Sci., Part A-2, 9, 1747 (1971).

7. R. S. Stein and T. Hashimoto, J. Polym. Sci., Part A-2, 9, 517 (1971).

8. D. Y. Yoon and R.S. Stein, J. Polym. Sci., Polym. Phys. Ed., 12, 763 (1974).

9. R. S. Moore and C. Gieniewsky, J. Appl. Phys., 36, 3022 (1965).

10. R. S. Moore and C. Gieniewsky, J. Polym. Sci., Part C, 13, 55 (1966).

11. J. J. van Aartsen and R. S. Stein, J. Polym. Sci., Part B, 5, 901 (1967).

12. J. J. van Aartsen and R. S. Stein, J. Polym. Sci., Part A-2, 9, 295 (1967).

13. J. J. van Aartsen, ONR Technical Report No. 83, Project: NR 056-378, Contract. Nonr 3357(01), Polymer Research Institute, University of Massachusetts, 1966.

14. R. S. Stein and M. B. Rhodes, J. Appl. Phys., 31, 1873 (1960).

15. Z. W. Wilchinsky, Polymer, 5, 271 (1964).

16. K. Kobayashi and T. Nagasawa, J. Polym. Sci., Part C, 15, 163 (1966).

17. K. Kataoka, T. Takahashi, and I. Tsujimoto, Sen-I Gakkaishi, 28, 336 (1972).

18. T. Oda, N. Sakaguchi, and H. Kawai, J. Polym. Sci., Part C, 5, 223 (1966).

19. A. Todo, to be included in $\mathrm{Ph}$. D. thesis to be submitted to Kyoto University, Kyoto, Japan. 